\title{
REGULARITY OF NET SUMMABILITY TRANSFORMS ON CERTAIN LINEAR TOPOLOGICAL SPACES
}

\author{
R. H. COX AND R. E. POWELL
}

The famous Silverman-Toeplitz theorem concerning the regularity of a matrix transform $A$ with complex entries has undergone much study and many generalizations. Among these have been contributions by Kojima [5], Fraleigh [3], Adams [1], and Agnew [2] concerning the summability of multiple sequences. Melvin-Melvin [6] examined the case where each entry in $A$ is a bounded linear operator on a Banach space. Recently Ramanujan [7] extended this idea to two types of linear topological spaces; namely, Fréchet spaces and locally bounded spaces. It is the purpose of this paper to generalize the results of Ramanujan by allowing the "rows" and "columns" of $A$ to be nets of linear continuous transformations and to investigate conditions under which $A$ will transform certain convergent nets into convergent nets.

We shall require the following notation and assumptions:

$X, Y:$ linear spaces over the complex numbers;

$\Re$ : a collection of seminorms (see [8] for definition) on $X$ which separates points of $X$ (i.e., if $x \in X-\{0\}$, then there is an $N \in \Re$ such that $N(x)>0$ );

TT: a collection of seminorms on $Y$ that separates points;

$\mathcal{F}$ : the locally convex, Hausdorff topology on $X$ generated by $\Re$;

G: the locally convex, Hausdorff topology on $Y$ generated by $\mathfrak{T}$;

$\{D, \leqq\}:$ a directed set with finite initial segments (i.e., if $d \in D$, then the set $\{e \mid e \in D$ and $e \leqq d\}$ is finite);

$C$ : the collection of all bounded convergent nets from $D$ to $X$. In this context, a net will be said to be convergent provided it has a limit. For a general discussion of nets, see [4]. If $f \in C$ and $N \in \Re$, then let $N^{c}(f)=\sup \{N(f(d)) \mid d \in D\}$. $\Re^{c}$ : the set of all $N^{c}$, for $N \in \Re$;

$\mathcal{F}^{c}$ : the locally convex, Hausdorff topology on $C$ generated by $\Re^{\circ}$.

We shall assume, for the remainder of the paper, that the linear topological space $\left\{C, F^{c}\right\}$ is barrelled (see [8] for definition). This

Received by the editors July 12, 1968. 
condition is necessary and sufficient (so long as $Y$ is nondegenerate) to insure that the following version of the Uniform Boundedness Principle holds. For a proof and related results, see Chapter IV of [8]; and, particularly, Theorem 3 on page 69.

THEOREM 1. If $J$ is a collection of linear continuous operators from $\left\{C, \Im^{c}\right\}$ to $\{Y, \varsigma\}$ with the property

$$
\text { if } f \in C \text { and } M \in \mathfrak{N} \text {, then }\{M(T(f)) \mid T \in \Im\} \text { is bounded; }
$$

then, for each $M \in \mathfrak{M}$, there is a finite subset $\left\{N_{i}^{c}\right\}_{i=1}^{n}$ of $\Re^{c}$ and a positive number $K$ such that

$$
\text { if } f \in C \text { and } T \in \mathcal{J} \text {, then } M(T(f)) \leqq K \sup _{l \leqq i \leqq_{n}} N_{i}^{e}(f) .
$$

Observe that, if $\mathscr{N}$ is countable and $\{X, \mathscr{F}\}$ is complete, then $\left\{C, F^{c}\right\}$ is second category and thus barrelled. That is, if $\{X, \mathcal{F}\}$ is a Fréchet space then $\left\{C, \mathscr{F}^{c}\right\}$ is barrelled.

Let $A$ be a function from $D \times D$ into the set of linear continuous functions from $\{X, \mathcal{F}\}$ to $\{Y, \mathcal{S}\}$ (denoted hereafter by $\mathcal{L}(X, Y)$ ). The $D \times D$ matrix $A$ is said to be convergence preserving provided that, if $f \in C$ and $d \in D$, then the net $h(e)=\sum_{e^{\prime} s o} A\left(d, e^{\prime}\right) f\left(e^{\prime}\right)$ is bounded and convergent in $\{Y, \rho\}$; and the net $A(f)$ defined, for $d \in D$, by $A(f)(d)=\lim _{e} h(e)$ is also bounded and convergent in $\{Y, \mathcal{g}\}$. Further, if $L \in \mathfrak{L}(X, Y)$, then $A$ is said to be $L$-regular provided $A$ is convergence preserving and, if $f \in C$, then $A(f)$ has limit $L\left(\lim _{d} f(d)\right)$.

The main result of this paper is the following Toeplitz theorem which generalizes Theorem 1 of [7].

Theorem 2. Suppose $\{Y, g\}$ is complete-in the sense that every Cauchy net from $D$ to $Y$ is a convergent net. The function $A$ is convergence preserving if, and only if, the following four statements are true:

(1) For each $M \in \mathfrak{N}$, there is a finite subset $\left\{N_{i}^{c}\right\}_{i=1}^{n}$ of $\Re^{c}$ and a positive number $K$ such that, if $f \in C$ and $(d, e) \in D \times D$, then

$$
M\left(\sum_{e^{\prime} \leq e} A\left(d, e^{\prime}\right) f\left(e^{\prime}\right)\right) \leqq K \sup _{l \leqq i \leq_{n}} N_{i}^{e}(f) .
$$

(2) If $f \in C$ and $d \in D$, then the net $h(e)=\sum_{e^{\prime} s e} A\left(d, e^{\prime}\right) f\left(e^{\prime}\right)$ is convergent in $\{Y, g\}$.

(3) If $f \in C$ with limit 0 and $d \in D$ then the net defined, for $d_{0} \in D$, by

$$
g\left(d_{0}\right)=\lim _{e} \sum_{e^{\prime} \leqslant e_{i} d \ddagger e^{\prime}} A\left(d_{0}, e^{\prime}\right) f\left(e^{\prime}\right),
$$

is convergent in $\{Y, \mathrm{~g}\}$. 
(4) If $x \in X$, then the net defined, for $d \in D$, by

$$
k(d)=\lim _{e} \sum_{e^{\prime} \leq e} A\left(d, e^{\prime}\right)(x),
$$

is convergent in $\{Y, \mathrm{~g}\}$.

Proof. Suppose that $A$ is convergence preserving. For each pair $(d, e)$ in $D \times D$, let $T_{(d, e)}$ be the linear function from $C$ to $Y$ defined, for $f \in C$, by $T_{(d, e)}(f)=\sum_{e^{\prime} \xi e} A\left(d, e^{\prime}\right) f\left(e^{\prime}\right)$. Since $D$ has finite initial segments and $A$ has entries in $\mathfrak{L}(X, Y)$, it is easily seen that $T_{(d, e)}$ is continuous from $\left\{C, \mathfrak{F}^{c}\right\}$ to $\{Y, \mathcal{S}\}$. Furthermore, if $d \in D$ and $f \in C$, then the net $h(e)=T_{(d, e)}(f)$ is bounded in $\{Y, q\}$. Suppose $M \in \mathfrak{N}$ and $d \in D$. By Theorem 1 , there is a finite subset $\left\{N_{i}^{c}(f)\right\}_{i=1}^{n}$ in $\Re^{c}$ and a positive number $K$ such that, if $e \in D$ and $f \in C$, then

$$
M\left(T_{(d, e)}(f)\right) \leqq K \sup _{1 \leqq i \geqq n} N_{i}^{c}(f) .
$$

Thus, if $f \in C$,

$$
M\left(\lim _{\bullet} T_{(d, e)}(f)\right) \leqq K \sup _{1 \leqq i \leqq n} N_{i}^{c}(f)
$$

and the linear function defined, for $f \in C$, by $T_{d}(f)=\lim _{e} T_{(d, e)}(f)$ is continuous from $\left\{C, F^{c}\right\}$ to $\{Y, g\}$. Finally, if $f \in C$, then the net $k(d)=T_{d}(f)$ is bounded in $\{Y, \mathcal{G}\}$ and a reapplication of Theorem 1 shows that statement (1) holds. Statement (2) is immediate from the definition. Concerning statement (3), suppose $d \in D$ and $f \in C$. If $e \in D$, let

$$
\begin{aligned}
f^{\prime}(e) & =f(e) & & \text { if } d \pm e, \\
& =0 & & \text { if } d<e .
\end{aligned}
$$

Then $f^{\prime} \in C$ has limit 0 ; and, if $\left(d_{0}, e\right) \in D \times D$,

$$
\sum_{e^{\prime} \leq e} A\left(d_{0}, e^{\prime}\right) f^{\prime}\left(e^{\prime}\right)=\sum_{e^{\prime} \leq e ; d \neq e^{\prime}} A\left(d_{0}, e^{\prime}\right) f\left(e^{\prime}\right) .
$$

Statement (3) now follows. Finally, let $x \in X$ and define, for $d \in D$, $f(d)=x$. Thus $f \in C$ and statement (4) follows from statement (2) and the definition.

To prove the converse, suppose that statements (1), (2), (3) and (4) hold; $M \in \mathscr{T}, \epsilon>0$, and $f \in C$. Let $\left\{N_{i}^{c}\right\}_{i=1}^{n}$ be a finite subset of $\Re^{c}$ and $K$ a positive number with the properties of statement (1). Let $d_{0} \in D$ be such that, if $d \in D$ and $d_{0} \leqq d$, then $N_{i}(f(d)-\lim f)<\epsilon / 4 K$ for $i=1,2, \cdots, n$. Secondly, let $d_{1} \in D$ be such that, if $d \in D$ and $d_{1} \leqq d$, then 
(a) $M\left(\lim _{e} \sum_{e^{\prime} \leq e} A\left(d, e^{\prime}\right)(\lim f)-\lim _{e} \sum_{e^{\prime} \leq e} A\left(d_{1}, e^{\prime}\right)(\lim f)\right)<\frac{\epsilon}{4}$.

and

(b) $M\left(\lim _{e} \sum_{e^{\prime} \leqq e ; d_{0 \neq e^{\prime}}} A\left(d, e^{\prime}\right)\left(f\left(e^{\prime}\right)-\lim f\right)\right.$

$$
\left.-\lim _{e} \sum_{e^{\prime} \leqq e ; d_{0} \ddagger e^{\prime}} A\left(d_{1}, e^{\prime}\right)\left(f\left(e^{\prime}\right)-\lim f\right)\right)<\frac{\epsilon}{4} .
$$

If $d \in D$ and $d_{1} \leqq d$, then

$$
M\left(\lim _{e} \sum_{e^{\prime} \leq e} A\left(d, e^{\prime}\right) f\left(e^{\prime}\right)-\lim _{\bullet} \sum_{e^{\prime} \leq e} A\left(d_{1}, e^{\prime}\right) f\left(e^{\prime}\right)\right)<\epsilon .
$$

Thus, since $\{Y, g\}$ is net complete, $A$ is convergence preserving and Theorem 2 is proved.

An analogous argument will yield a proof of the following theorem where $L \in \mathcal{L}(X, Y)$.

THEOREM 3. The function $A$ is L-regular if, and only if, statements (1) and (2) of Theorem 2 hold and the following two statements also hold:

(3') If $f \in C$ with limit 0 and $d \in D$ then the net defined, for $d_{0} \in D$, by

$$
g\left(d_{0}\right)=\lim _{e} \sum_{e^{\prime} \leqq e ; d \xi e^{\prime}} A\left(d_{0}, e^{\prime}\right) f\left(e^{\prime}\right),
$$

is convergent to 0 in $\{Y, \mathrm{~g}\}$.

(4') If $x \in X$, then the net defined, for $d \in D$, by

$$
k(d)=\lim _{e} \sum_{e^{\prime} \leq e} A\left(d, e^{\prime}\right)(x),
$$

is convergent to $L(x)$ in $\{Y, \mathcal{G}\}$.

It should be noted that in Theorem 3 we need not require that $\{Y, \mathcal{G}\}$ be net complete.

We see that Theorems 2 and 3 are extensions of Theorems 1 and 2 of [7], as well as the usual Silverman-Toeplitz theorems. However, there is one application which is important in the study of functions of several complex variables that is included in the present theory but not included in [7]. In particular, suppose that each of $\{X, \mathcal{F}\}$ and $\{Y, \varsigma\}$ is the complex plane with the usual topology, $k$ is a positive integer, and $D$ is the set of $k$-tuples of positive integers where, if each of $n$ and $m$ is in $D$, then $n<m$ provided that, for $i=1,2, \cdots, k$, $n(i)<m(i)$. In this case, letting $I$ denote the identity function on $X$, 
we have the following theorem (of course, in this setting, $A$ is simply a $D \times D$ matrix of complex numbers).

Theorem 4. The function $A$ is I-regular if, and only if, the following three statements are true:

(1) There is a positive number $K$ such that, if $(d, e) \in D \times D$, then $\sum_{e^{\prime} \leq e}\left|A\left(d, e^{\prime}\right)\right| \leqq K$.

(2) If $d \in D$, then the complex number net defined, for $d_{0} \in D$, by

$$
g\left(d_{0}\right)=\lim _{e} \sum_{e^{\prime} \leqq e ; d \neq e^{\prime}}\left|A\left(d_{0}, e^{\prime}\right)\right|
$$

is convergent to 0 .

(3) The net defined, for $d \in D, b y$

$$
k(d)=\lim _{e} \sum_{e^{\prime} \leq e} A\left(d, e^{\prime}\right)
$$

has limit 1.

Proof. In this context, statements (1) and (2) of Theorem 2 are equivalent to statement (1). Also statement $\left(4^{\prime}\right)$ of Theorem 3 is equivalent to statement (3). As statement (2) implies statement ( $\left.3^{\prime}\right)$ of Theorem 3, all that remains is to show that if $A$ is $I$-regular, then statement (2) holds. Hence, suppose that $A$ is $I$-regular, $d \in D$; and for each $d_{0} \in D$, let

$$
g\left(d_{0}\right)=\lim _{\bullet} \sum_{e^{\prime} \leq e ; d \neq e^{\prime}}\left|A\left(d_{0}, e^{\prime}\right)\right| .
$$

Suppose further that $g$ does not have limit 0 , and let $\epsilon>0$ be such that, if $e \in D$, then there is an $>e^{\prime}$ with the property that $g\left(e^{\prime}\right)>\epsilon$. Let $S=\left\{d^{\prime} \mid d^{\prime} \in D, d d^{\prime}\right\}, e_{1} \in D$ such that $e_{1}>1$ (the constant 1 member of $D$ ) and $K_{1}$ a finite subset of $S$ such that

$$
\sum_{e \in S}\left|A\left(e_{1}, e\right)\right|-\sum_{e \in K_{1}}\left|A\left(e_{1}, e\right)\right|<\frac{\epsilon}{4} .
$$

Suppose $p$ is a positive integer and disjoint finite subsets $K_{1}, K_{2}$, $\cdots K_{p}$ of $S$ have been chosen, along with elements $e_{1}, e_{2}, \cdots, e_{p}$ of $D$. Using the fact that if $e \in D$, then the net $A(\cdot, e)$ has limit 0 ; let $e_{p+1} \in D$ be such that

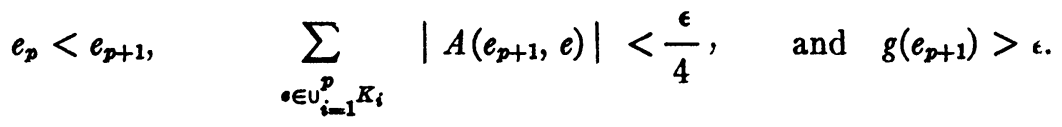

Let $K_{p+1}$ be a finite subset of $S$ such that $K_{p+1} \cap \bigcup_{i=1}^{p} K_{i}=\varnothing$ and 


$$
\sum_{e \in S}\left|A\left(e_{p+1}, e\right)\right|-\sum_{e \in K_{p+1}}\left|A\left(e_{p+1}, e\right)\right|<\frac{\epsilon}{4} .
$$

Define the member $f$ of $C$ as follows: if $e \in D$, then

$$
\begin{aligned}
f(e) & =\left|A\left(e_{n}, e\right)\right| / A\left(e_{n}, e\right) \quad \text { if } e \in K_{n} \quad \text { and } \quad A\left(e_{n}, e\right) \neq 0, \\
& =0, \quad \text { otherwise. }
\end{aligned}
$$

If $p$ is a positive integer, then

$$
\begin{aligned}
\left|\lim _{\boldsymbol{e}} \sum_{e^{\prime} \leqq e} A\left(e_{p}, e^{\prime}\right) f\left(e^{\prime}\right)\right| & \geqq \sum_{e^{\prime} \in K_{p}}\left|A\left(e_{p}, e^{\prime}\right)\right|-\sum_{e^{\prime} \in S-K_{p}}\left|A\left(e_{p}, e^{\prime}\right)\right| \\
& =\sum_{e^{\prime} \in S}\left|A\left(e_{p}, e^{\prime}\right)\right|-2 \sum_{e^{\prime} \in S-K_{p}}\left|A\left(e_{p}, e^{\prime}\right)\right| \\
& >\epsilon-\epsilon / 2=\epsilon / 2 .
\end{aligned}
$$

Since the sequence $e$ is cofinal in $D$, we see that $A(f)$ does not have limit 0 . This contradiction establishes Theorem 4 .

\section{BIBLIOGRAPHY}

1. C. R. Adams, On summability of double series, Trans. Amer. Math. Soc. 34 (1932), 215-230.

2. R. P. Agnew, On summability of multiple sequences, Amer. J. Math. 56 (1934), 62-68.

3. P. A. Fraleigh, Regular bilinear transformations of sequences, Amer. J. Math. 53 (1931), 697-709.

4. J. L. Kelly, General topology, Van Nostrand, Princeton, N. J., 1955.

5. T. Kojima, On the theory of double sequences, Tôhoku Math. J. 21 (1922), 3-14.

6. H. Melvin-Melvin, On generalized K-transformations in Banach spaces, Proc. London Math. Soc. 53 (1951), 83-108.

7. M. S. Ramanujan, Generalized Kojima-Toeplitz matrices in certain linear topological spaces, Math. Ann. 159 (1965), 365-373.

8. A. P. Robertson and W. J. Robertson, Topological vector spaces, Cambridge Univ. Press, New York, 1964.

UNIVERSITY OF KENTUCKY 